\title{
Snail and serpinA1 promote tumor progression and predict prognosis in colorectal cancer
}

\author{
Chae Hwa Kwon ${ }^{1, *}$, Hye Ji Park ${ }^{1, *}$, Jin Hwa Choi ${ }^{1}$, Ja Rang Lee ${ }^{1}$, Hye Kyung Kim ${ }^{1}$, \\ Hong-jae $\mathrm{Jo}^{2}$, Hyun Sung Kim², Nahmgun Oh${ }^{2}$, Geun Am Song ${ }^{3}$ and Do Youn Park ${ }^{1}$ \\ ${ }^{1}$ Department of Pathology, Pusan National University Hospital and Pusan National University School of Medicine, and \\ BioMedical Research Institute, Pusan National University Hospital, Seo-Gu, Busan, Korea \\ ${ }^{2}$ Department of Surgery, Pusan National University Hospital and Pusan National University School of Medicine, and BioMedical \\ Research Institute, Pusan National University Hospital, Seo-Gu, Busan, Korea \\ ${ }^{3}$ Department of Internal Medicine, Pusan National University Hospital and Pusan National University School of Medicine, and \\ BioMedical Research Institute, Pusan National University Hospital, Seo-Gu, Busan, Korea \\ * These authors have contributed equally to this work
}

Correspondence to: Do Youn Park, email: pdy220@pusan.ac.kr

Keywords: colorectal cancer, snail, serpinA 1, prognosis, fibronectin

Received: December 16, $2014 \quad$ Accepted: April 10, 2015

Published: April 29, 2015

This is an open-access article distributed under the terms of the Creative Commons Attribution License, which permits unrestricted use, distribution, and reproduction in any medium, provided the original author and source are credited.

\section{ABSTRACT}

The role of Snail and serpin peptidase inhibitor clade A member 1 (serpinA1) in tumorigenesis has been previously identified. However, the exact role and mechanism of these proteins in progression of colorectal cancer (CRC) are controversial. In this study, we investigated the role of Snail and serpinA1 in colorectal cancer (CRC) and examined the mechanisms through which these proteins mediate CRC progression. Immunohistochemical analysis of $\mathbf{5 2 8}$ samples from patients with CRC showed that elevated expression of Snail or serpinA1 was correlated with advanced stage, lymph node metastasis, and poor prognosis. Moreover, we detected a correlation between Snail and serpinA1 expression. Functional studies performed using the CRC cell lines DLD-1 and SW-480 showed that overexpression of Snail or serpinA1 significantly increased CRC cell invasion and migration. Conversely, knockdown of Snail or serpinA1 expression suppressed CRC cell invasion and migration. ChIP analysis revealed that Snail regulated serpinA1 by binding to its promoter. In addition, fibronectin mediated Snail and serpinA1 signaling was involved in CRC cell invasion and migration. Taken together, our data showed that Snail and serpinA1 promoted CRC progression through fibronectin. These findings suggested that Snail and serpinA1 were novel prognostic biomarkers and candidate therapeutic targets in CRC.

\section{INTRODUCTION}

Snail is a family of zinc-finger transcription factors implicated in induction of the epithelial-mesenchymal transition (EMT) via suppression of E-cadherin expression, which disrupts normal epithelial cellcell adhesion and facilitates invasion [1-3]. Enhanced expression of Snail has been found in a variety of cancer types, including breast, ovarian, prostate, lung, and gastric cancers, as well as melanoma, and has been reported to be frequently associated with invasiveness, metastasis, and poor prognosis [4-10].
In colorectal cancer (CRC), Snail expression is significantly elevated $[11,12]$ and has been reported to promote cancer progression by suppressing 15PGDH expression [13]. Snail is also associated with downregulation of the vitamin $\mathrm{D}$ receptor and E-cadherin in CRC [14]. However, the tumorigenic effects and underlying mechanisms through which Snail mediates $\mathrm{CRC}$ are still not completely understood. Moreover, the results of clinical investigations are controversial. Roy et al. [11] and Pena et al. [14] have reported that the aberrant expression of Snail in tumors may be associated with metastatic ability, whereas Kroepil et al. [12] showed that 
there is no significant correlation between Snail expression in tumors and clinicopathological parameters or overall survival. Despite these findings, the clinical significance of Snail expression in CRC remains poorly understood.

Previously, we observed that Snail was correlated with prognosis in gastric cancer [15]. We have also reported that serpin peptidase inhibitor clade A member 1 (serpinA1) is a direct target of Snail in gastric cancer cells. SerpinA1, a type of serine protease inhibitor, has been reported to modulate invasive and metastatic capacity in lung cancer, gastric cancer, and CRC [16-18]. Moreover, serpinA1 mRNA expression is elevated in blood samples from patients with CRC patients and is an accurate biomarker for predicting prognoses in patients with CRC [19]. However, the mechanisms through which serpinA1 promote CRC progression are not yet known.

In this study, we identified Snail and serpinA1 as prognostic biomarkers of poor overall survival in CRC. In addition, we demonstrated that Snail and serpinA1 were important regulators of CRC cell invasion and migration through a pathway involving upregulation of fibronectin. Thus, our study provides important insights into the mechanisms of CRC pathogenesis.

\section{RESULTS}

\section{Snail and serpinA1 were prognostic factors for patients with CRC}

To assess whether Snail and serpinA1 were involved in the progression of CRC, we first conducted immunohistochemical staining for Snail and serpinA1 in 528 and 522 CRC tissues, respectively, and analyzed the clinical relevance of Snail and serpinA1 expression. For Snail, only detectable nuclear staining was considered positive. Positive nuclear staining signals for Snail at levels of less than $75 \%$ and greater than or equal to $75 \%$ were observed in $73.7 \%(389 / 528)$ and $26.3 \%$ (139/528) of cases, respectively (Figure 1A and 1B). For serpinA1, cytoplasmic staining in tumor cells was considered positive. Negative (-) and low (+) or strong (++) cytoplasmic staining for serpinA1 were noted in $65.1 \%$ $(340 / 522)$ and $34.9 \%(182 / 522)$ of cases, respectively (Figure 1C-1E). Furthermore, there was a significant positive correlation between the expression of Snail and serpinA1 $(P<0.0001, r=0.217$; data not shown).

Snail was overexpressed ( $\geq 75 \%$ positivity) in
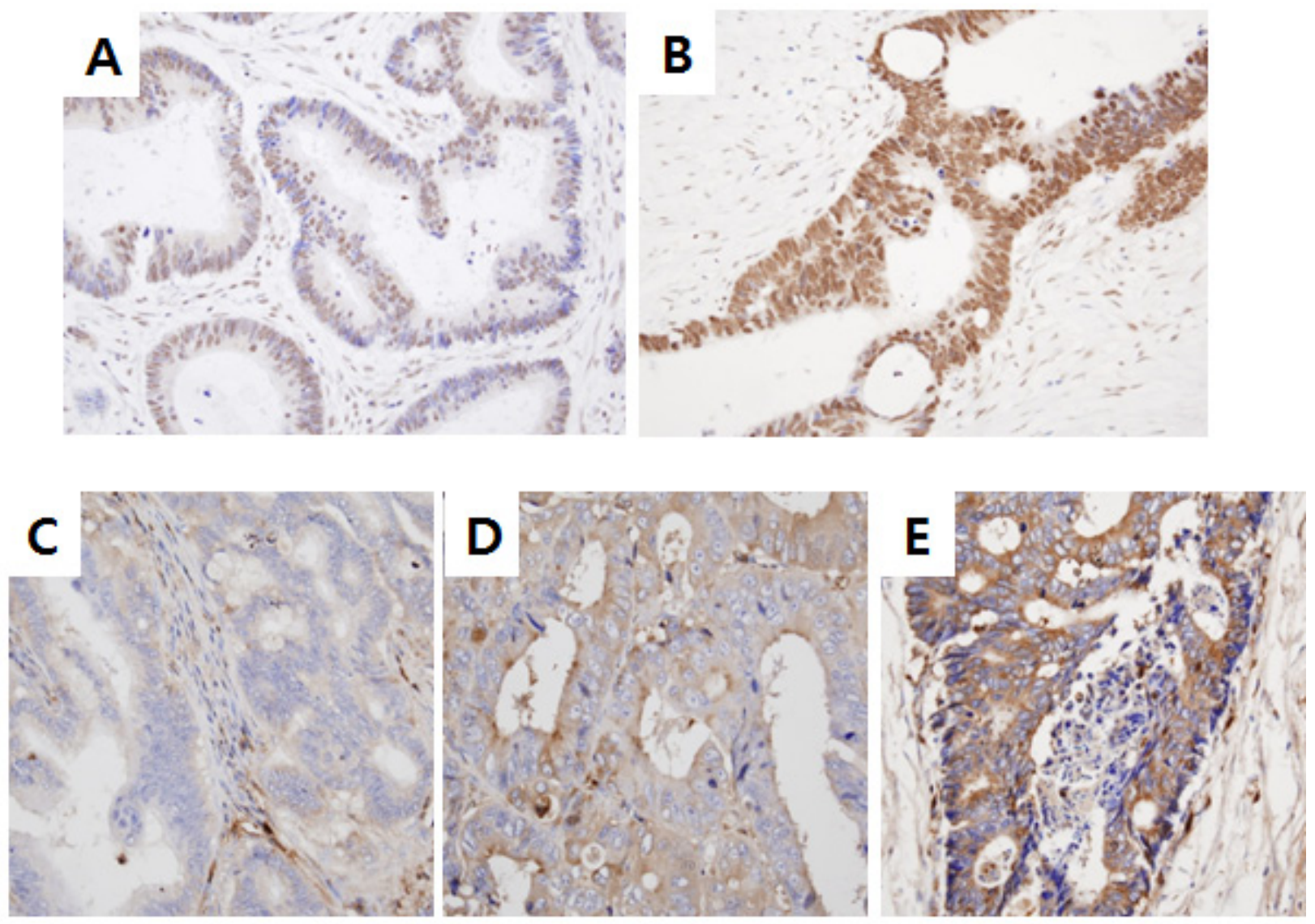

Figure 1: Snail and serpinA1 expression in patients with CRC. Snail and serpinA1 expression levels were determined by IHC analysis in sections of TMAs. Weak $(<75 \%)$ A. or strong $(\geq 75 \%)$ B. nuclear Snail immunostaining was detected in tumor cells. Negative C., weak D., or strong $\mathbf{E}$. cytoplasmic staining for serpinA1 was observed in tumor cells. 
Table 1: Relationship between Snail expression and clinicopathological characteristics in 528 patients with colorectal cancer.

\begin{tabular}{|c|c|c|c|c|}
\hline & \multirow[b]{2}{*}{ [No.] } & \multicolumn{2}{|c|}{ Snail expression } & \multirow{2}{*}{$P$ value } \\
\hline & & $<75 \%$ & $\geq 75 \%$ & \\
\hline Age (years) & 528 & $63.0 \pm 0.56$ & $63.5 \pm 1.06$ & 0.633 \\
\hline Size $(\mathrm{cm})$ & 528 & $5.12 \pm 0.11$ & $5.38 \pm 0.19$ & 0.246 \\
\hline \multicolumn{5}{|l|}{ Sex } \\
\hline Male & 308 & $229(74.4)$ & $79(25.6)$ & \multirow{2}{*}{0.676} \\
\hline Female & 220 & $160(72.7)$ & $60(27.3)$ & \\
\hline \multicolumn{5}{|l|}{ Location } \\
\hline Right Colon & 129 & $91(70.5)$ & $38(29.5)$ & \multirow[t]{2}{*}{0.353} \\
\hline Left Colon & 399 & $298(74.7)$ & $101(25.3)$ & \\
\hline \multicolumn{4}{|l|}{ Histological type } & \multirow[t]{5}{*}{$<0.0001$} \\
\hline Well & 46 & $37(80.4)$ & 9 (19.6) & \\
\hline Moderately & 427 & $323(75.6)$ & $104(24.4)$ & \\
\hline Poorly & 29 & $11(37.9)$ & $18(62.1)$ & \\
\hline Mucinous & 26 & $18(69.2)$ & $8(30.8)$ & \\
\hline \multicolumn{4}{|l|}{ Invasion depth } & \multirow[t]{5}{*}{0.009} \\
\hline T1 & 25 & $23(92.0)$ & $2(8.0)$ & \\
\hline $\mathbf{T} 2$ & 75 & $60(80.0)$ & $15(20.0)$ & \\
\hline T3 & 374 & $274(73.3)$ & $100(26.7)$ & \\
\hline T4 & 54 & $32(59.3)$ & $22(40.7)$ & \\
\hline \multicolumn{4}{|l|}{ Perineural invasion } & \multirow{3}{*}{0.002} \\
\hline Negative & 330 & $258(78.2)$ & $72(21.8)$ & \\
\hline Positive & 198 & $131(66.2)$ & $67(33.8)$ & \\
\hline \multicolumn{5}{|l|}{ Lymphovascular } \\
\hline $\begin{array}{l}\text { emboli } \\
\text { Negative }\end{array}$ & 318 & $246(77.4)$ & $72(22.6)$ & \multirow[t]{2}{*}{0.018} \\
\hline Positive & 210 & $143(68.1)$ & $67(31.9)$ & \\
\hline \multicolumn{4}{|l|}{$\begin{array}{l}\text { Lymph node } \\
\text { metastasis }\end{array}$} & \multirow[t]{6}{*}{0.001} \\
\hline NO & 282 & $220(78.0)$ & $62(22.0)$ & \\
\hline N1a(1) & 72 & $55(76.4)$ & $17(23.6)$ & \\
\hline N1b(2-3) & 84 & $61(72.6)$ & $23(27.4)$ & \\
\hline N2a(4-6) & 49 & $34(69.4)$ & $15(30.6)$ & \\
\hline $\mathbf{N} 2 \mathbf{b}(\geq 7)$ & 41 & $19(46.3)$ & $22(53.7)$ & \\
\hline \multicolumn{4}{|l|}{ Microsatellite status } & \multirow[t]{4}{*}{0.486} \\
\hline MSS & 466 & $340(73.0)$ & $126(27.0)$ & \\
\hline MSI-L & 19 & $14(73.7)$ & $5(26.3)$ & \\
\hline MSI-H & 43 & $35(81.4)$ & $8(18.6)$ & \\
\hline
\end{tabular}

Table 2: Multivariate survival analysis with Cox regression model in 528 colorectal cancers.

\begin{tabular}{|c|c|c|c|c|}
\hline Variables & B & SE & HR (95\% CI) & $P$ \\
\hline Age $(<63$ and $\geq 63)$ & -0.293 & 0.175 & $0.746(0.530-1.050)$ & 0.093 \\
\hline $\begin{array}{lll}\begin{array}{l}\text { Lymph } \\
\text { (absent } v s . \text { present) }\end{array} & \text { metastasis } \\
\end{array}$ & -1.114 & 0.172 & $0.328(0.227-0.475)$ & $<0.0001$ \\
\hline Gender (male vs. female) & -0.001 & 0.170 & $0.999(0.716-1.393)$ & 0.993 \\
\hline Site (right $v s$. left colon) & 0.241 & 0.188 & $1.272(0.881-1.838)$ & 0.199 \\
\hline $\begin{array}{ll}\begin{array}{l}\text { Depth } \\
(\mathrm{T} 1, \mathrm{~T} 2 \text { vs. T3,T4) }\end{array} & \text { invasion } \\
\end{array}$ & -0.625 & 0.310 & $0.536(0.292-0.983)$ & 0.044 \\
\hline Snail $(<75 \%$ and $\geq 75 \%)$ & -0.678 & 0.172 & $0.507(0.362-0.712)$ & $<0.0001$ \\
\hline
\end{tabular}

Note: B, coefficient; HR, hazard ratio; CI, confidence interval 
poorly differentiated CRC $(P<0.0001$, Table 1$)$, and the expression of Snail was also strongly elevated in patients with advanced stages $(P=0.009)$, perineural invasion $(P$ $=0.002)$, lymphovascular emboli $(P=0.018)$, and lymph node metastasis $(P=0.001)$. Moreover, patients with at least $75 \%$ positive expression of Snail had significantly poorer survival rates than patients with less than $75 \%$ low expression $(P<0.0001$, Figure 2A). On the basis of multivariate Cox regression analysis, the expression of Snail was an independent prognostic factor for overall survival $(P<0.0001$, Table 2$)$. Lymph node metastasis was also shown to be an independent prognostic factor for overall survival $(P<0.0001$, Table 2$)$.

SerpinA1 overexpression was correlated with mean tumor size $(P=0.014$, Table 3$)$. SerpinA1 was also frequently overexpressed in patients with advanced stages $(P=0.014)$ and lymph node metastasis $(P=0.006)$. Moreover, Kaplan-Meier survival analysis showed a strong correlation between serpinA1 expression and shorter overall survival $(P=0.023$, Figure $2 \mathrm{~B})$. Taken together, these results showed that Snail and serpinA1 were associated with advanced clinical stage, lymph node metastasis, and poor prognosis in patients with CRC.

\section{Snail and serpinA1 induced CRC cell invasion and migration}

Next, we tested whether the Snail/serpinA1 signaling pathway was involved in the progression of CRC. DLD-1 and SW-480 cells were transfected with a Snail expression construct or siRNA against Snail (siSnail) for evaluation of the effects of Snail on invasion and migration. The expression levels of Snail were assessed by real-time PCR (Figure 3A and 3D). Our results showed that overexpression of Snail significantly increased invasion and migration in DLD-1 and SW-480 cells (Figure 3B and 3C). In contrast, invasion and migration were decreased by Snail knockdown (Figure 3E and 3F).

Similar experiments were performed to assess the role of serpinA1 in the progression of CRC. The expression levels of serpinA1 were analyzed by realtime PCR (Figure 4A and 4D). Our results showed that overexpression of serpinA1 also significantly increased the invasion and migration of DLD-1 and SW-480 cells (Figure 4B and 4C). Conversely, invasion and migration were decreased by serpinA1 knockdown (Figure 4E and 4F). These results suggested that Snail and serpinA1 were essential for conferring cancer-related phenotypes in DLD-1 and SW-480 cells.

\section{Snail regulated serpinA1 by binding to its promoter in CRC}

Since we had observed that Snail expression was correlated with serpinA1 expression in immunohistochemistry analysis, we investigate whether Snail could regulate serpinA1 expression. DLD-1 and SW-480 cells were transfected with Snail or serpinA1 expression constructs or siRNA targeting Snail or serpinA1. Overexpression of Snail increased serpinA1 expression, while knockdown of Snail decreased serpinA1 expression (Figure 5A). However, alteration of serpinA1 levels did not affect Snail expression (Figure 5B). These results suggested that serpinA1 may be regulated by Snail.
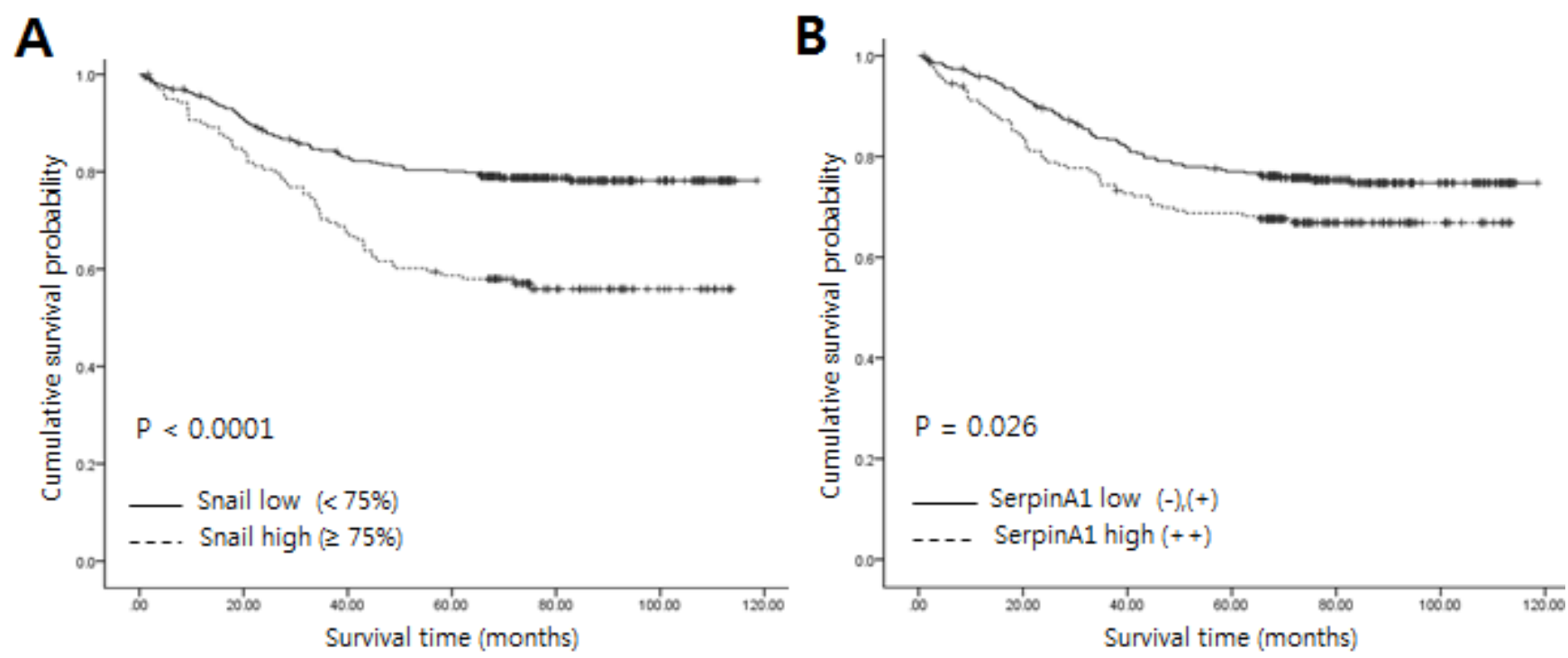

Figure 2: Relationship between expression of Snail or serpinA1 and clinical outcomes in patients with CRC. KaplanMeier survival analysis was performed according to Snail or serpinA1 expression. A., B. Linear relationships were observed between increased Snail or serpinA1 expression and shortened overall survival in CRC $(P<0.0001$ and $P=0.023$, respectively). $P$-values were calculated by the log-rank test. 
Table 3: Relationship between SerpinA1 expression and clinicopathological characteristics in 522 patients with colorectal cancer.

\begin{tabular}{|c|c|c|c|c|}
\hline & \multirow[b]{2}{*}{ [No.] } & \multicolumn{2}{|c|}{ Snail expression } & \multirow{2}{*}{$P$ value } \\
\hline & & $(-)(+)$ & $(++)$ & \\
\hline Age (years) & 522 & $62.8 \pm 0.62$ & $63.6 \pm 0.84$ & 0.434 \\
\hline Size (cm) & 522 & $5.02 \pm 0.12$ & $5.54 \pm 0.17$ & 0.014 \\
\hline \multicolumn{5}{|l|}{ Sex } \\
\hline Male & 303 & $197(65.0)$ & $106(35.0)$ & \multirow{2}{*}{0.947} \\
\hline Female & 219 & $143(65.3)$ & $76(34.7)$ & \\
\hline \multicolumn{5}{|l|}{ Location } \\
\hline Right Colon & 126 & $86(68.3)$ & $40(31.7)$ & \multirow[t]{2}{*}{0.399} \\
\hline Left Colon & 396 & $254(64.1)$ & $142(35.9)$ & \\
\hline Histological type & & & & \multirow[t]{5}{*}{0.269} \\
\hline Well & 45 & $30(66.7)$ & $15(33.3)$ & \\
\hline Moderately & 422 & $275(65.2)$ & $147(34.8)$ & \\
\hline Poorly & 29 & $15(51.7)$ & $14(48.3)$ & \\
\hline Mucinous & 26 & $20(76.9)$ & $8(23.1)$ & \\
\hline Invasion depth* & & & & \multirow[t]{5}{*}{0.014} \\
\hline T1 & 24 & $18(75.0)$ & $8(25.0)$ & \\
\hline $\mathbf{T} 2$ & 75 & $57(76.0)$ & $18(24.0)$ & \\
\hline T3 & 369 & $234(63.4)$ & 135 (36.6) & \\
\hline T4 & 54 & $31(57.4)$ & $23(42.6)$ & \\
\hline \multicolumn{4}{|l|}{ Perineural invasion } & \multirow{3}{*}{0.206} \\
\hline Negative & 326 & $219(67.2)$ & $107(32.8)$ & \\
\hline Positive & 196 & $121(61.7)$ & $75(38.3)$ & \\
\hline \multicolumn{5}{|l|}{$\begin{array}{l}\text { Lymphovascular } \\
\text { emboli }\end{array}$} \\
\hline Negative & 314 & $210(66.9)$ & $104(33.1)$ & \multirow[t]{2}{*}{0.304} \\
\hline Positive & 208 & $130(62.5)$ & $78(37.5)$ & \\
\hline $\begin{array}{l}\text { Lymph node } \\
\text { metastasis }\end{array}$ & & & & 0.006 \\
\hline N0 & 278 & $195(70.1)$ & $83(29.9)$ & \\
\hline $\mathrm{N} 1 \mathrm{a}(1)$ & 72 & $42(58.3)$ & $30(41.7)$ & \\
\hline$N 1 b(2-3)$ & 83 & $58(69.9)$ & $25(30.1)$ & \\
\hline N2a(4-6) & 48 & $26(54.2)$ & $22(45.8)$ & \\
\hline $\mathbf{N} 2 \mathbf{b}(\geq 7)$ & 41 & $19(46.3)$ & $22(53.7)$ & \\
\hline Microsatellite status & & & & 0.565 \\
\hline MSS & 460 & $296(64.3)$ & $164(35.7)$ & \\
\hline MSI-L & 19 & $14(73.7)$ & $5(26.3)$ & \\
\hline MSI-H & 43 & $30(69.8)$ & $13(30.2)$ & \\
\hline
\end{tabular}

\section{Note: *T1,T2 vs T3,T4,}

We previously reported that Snail regulates serpinA1 by binding to its promoter in gastric cancer [15]. Thus, to examine the molecular mechanisms through which Snail may regulate serpin $\mathrm{A} 1$ expression in $\mathrm{CRC}$, we performed ChIP assays using Snail-overexpressing cells. In these cells, there was more than a four-fold increase in the precipitation of the serpinA1 promoter with anti-Snail antibodies than with IgG alone (Figure 5C and 5D). These results indicated that Snail regulated serpinA1 by binding to its promoter.
Fibronectin mediated Snail and serpinA1 signaling in CRC

SerpinA1 has been reported to regulate the aggregation of fibronectin on surfaces of tumor cells, which may increase the probability of metastasis [21]. Thus, to further understand the mechanisms through which serpinA1 regulates CRC progression, we examined the effects of Snail and serpinA1 expression on fibronectin 
levels. Western blot analysis showed that the expression of fibronectin in DLD-1 and SW-480 cells was increased after transfection with Snail or serpinA1 expression constructs (Figure 6A). Conversely, knockdown of Snail or serpinA1 by transfection with the appropriate siRNA decreased the expression of fibronectin (Figure 6B). Importantly, alteration of fibronectin expression did not change levels of Snail or serpinA1 (Figure 6C).

Next, we checked whether fibronectin was also involved in the progression of $\mathrm{CRC}$. Invasion and migration were increased in cells transfected with the fibronectin expression construct (Figure 6D). Conversely, knockdown of fibronectin expression using specific siRNA attenuated cell invasion and migration (Figure 6E). These results suggested that fibronectin functioned downstream of Snail and serpinA1 signaling pathways and was necessary for CRC progression.
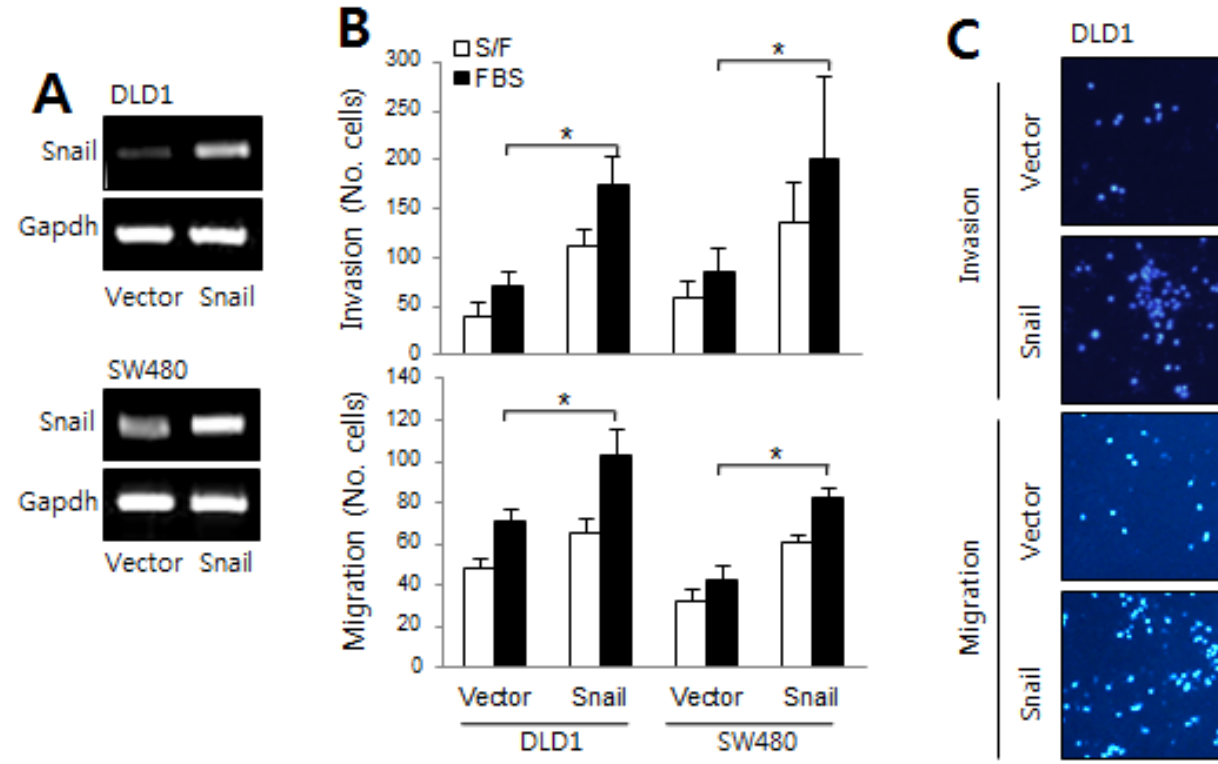

SW480
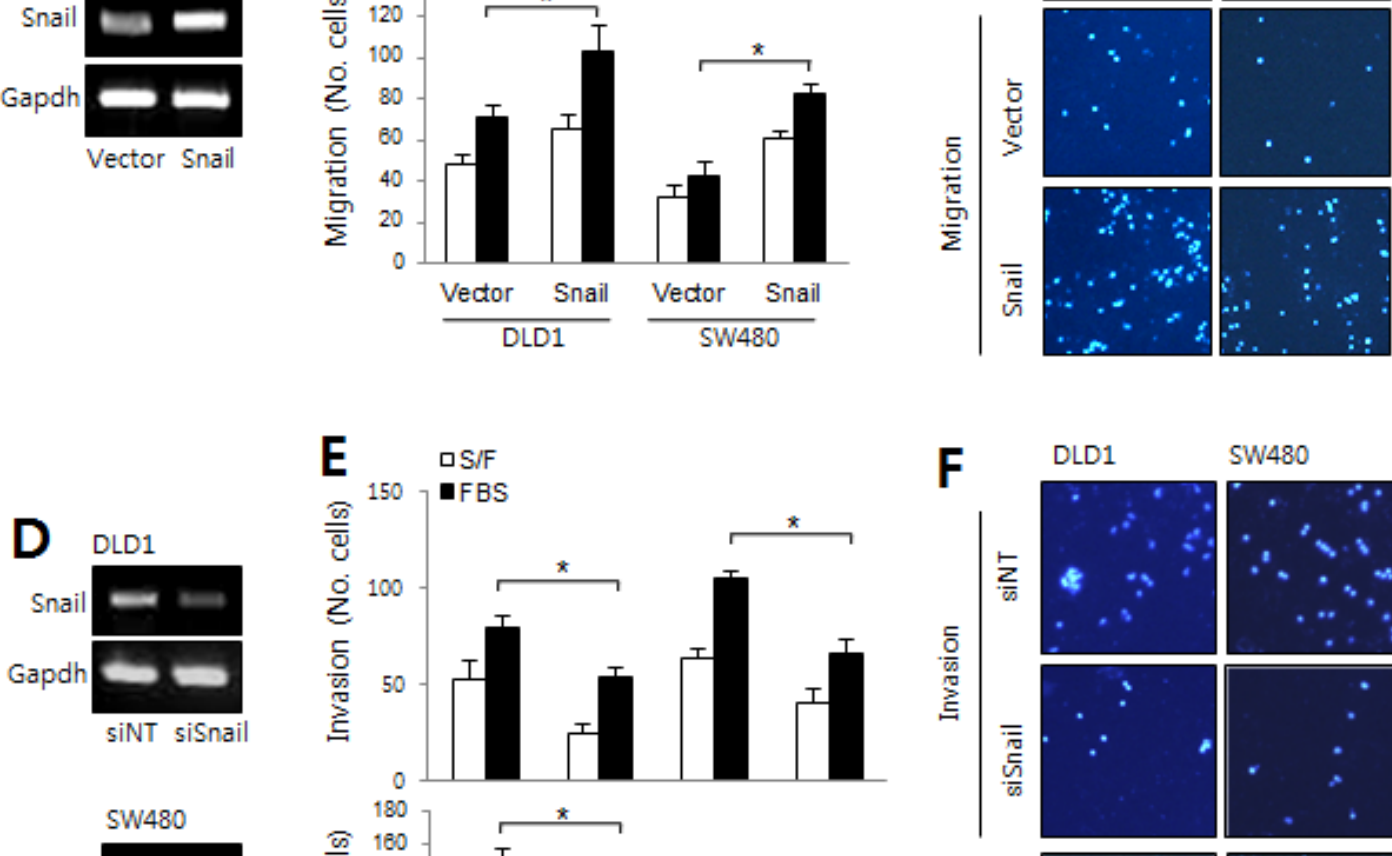

SW480
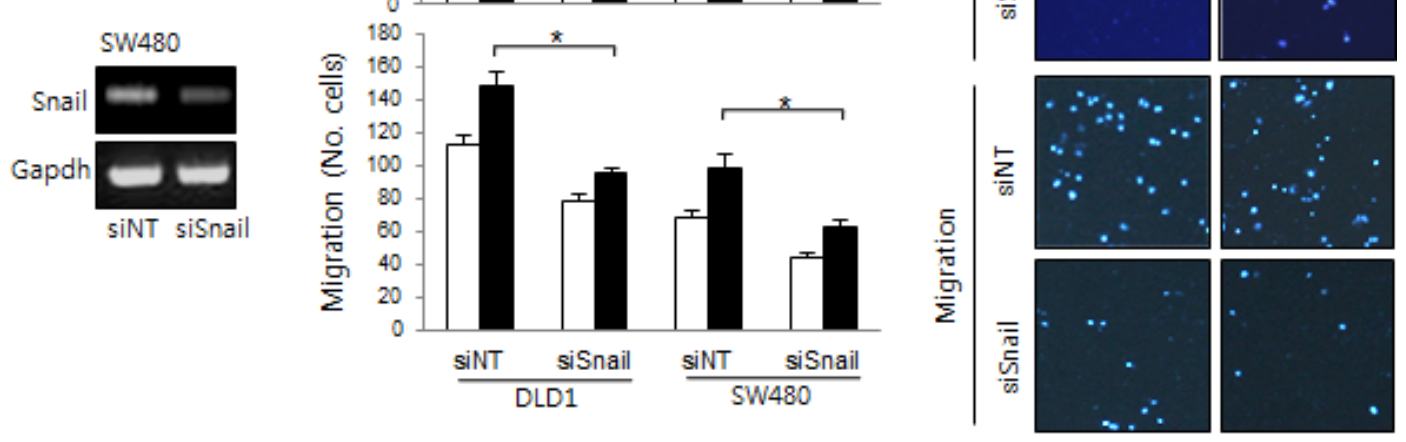

Figure 3: The roles of Snail in CRC. A. DLD-1 and SW480 cells were transfected with empty vector or a Snail expression construct to analyze migration and invasion capacity. Snail mRNA levels were determined by RT-PCR. B. Graphs show the number of cells that invaded (top) and migrated (bottom) in the presence or absence of $1 \%$ FBS. $* P<0.05$. C. Representative data are shown for cells that invaded (top) and migrated (bottom) in the presence of 1\% FBS. D. DLD-1 and SW480 cells were transfected with nontargeting siRNA (siNT) or Snail siRNA (siSnail), and migration and invasion assays were performed. Snail mRNA levels were then determined by RT-PCR. E. Graphs show the number of cells that invaded (top) and migrated (bottom) in the presence or absence of $1 \% \mathrm{FBS}$. $* P<0.05$. F. Representative data are shown for cells that invaded (top) and migrated (bottom) in the presence of $1 \%$ FBS. 


\section{The roles of Snail and serpinA1 in other types of human cancer cells}

Since Snail and serpinA1 are expressed not only in CRC but also in other types of cancer, [3, 16-18] we also examined the role of Snail and serpinA1 in breast and ovarian cancer cells (MCF7, MDA-MB-231, A2780, and SKVO3 cells) by modulating their expression. The results showed that Snail and serpinA1 induced migration in both breast and ovarian cancer cells (Figure 7A and 7B and Supplementary Figures 1 and 2). In addition, the results of ChIP assays showed that Snail may also modulate serpinA1 expression by binding to the serpinA1 promoter in breast and ovarian cancer cells (Figure 7C and 7D and Supplementary Figures 3). These results indicated that serpinA1 may be regulated by Snail and that Snail and serpinA1 signaling induces tumor progression in a variety of cancer cells.
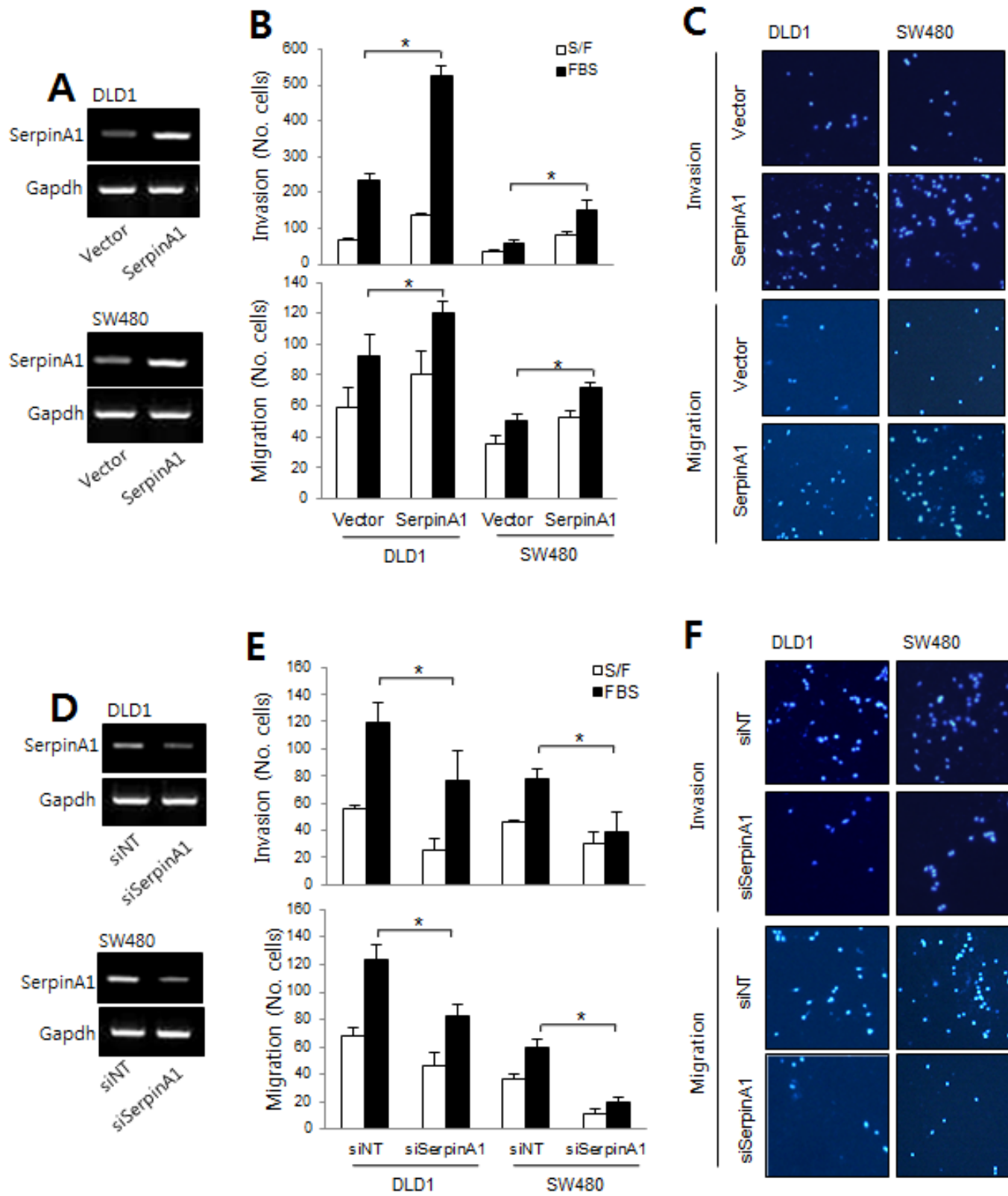

SW480

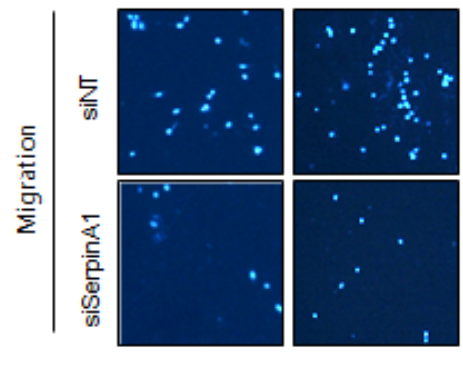

Figure 4: The roles of serpinA1 in CRC. A. DLD-1 and SW480 cells were transfected with empty vector or a serpinA1 expression construct, and migration and invasion assays were performed. SerpinA1 mRNA levels were determined by RT-PCR. B. Graphs show the number of cells that invaded (top) and migrated (bottom) in the presence or absence of $1 \%$ FBS. $* P<0.05$. C. Representative data are shown for cells that invaded (top) and migrated (bottom) in the presence of 1\% FBS. D. DLD-1 and SW480 cells were transfected with nontargeting siRNA (siNT) or serpinA1 siRNA (siSerpinA1) for the migration and invasion assays. SerpinA1 mRNA levels were determined by RT-PCR. E. Graphs show the number of cells that invaded (top) and migrated (bottom) in the presence or absence of $1 \%$ FBS. ${ }^{*} P<0.05$. F. Representative data are shown for cells that invaded (top) and migrated (bottom) in the presence of $1 \%$ FBS. 


\section{DISCUSSION}

CRC is a major cause of morbidity and mortality worldwide. The incidence of CRC in Korea has increased dramatically over the past few decades, while the incidence rates of more common cancers, such as stomach and liver cancers, have decreased [22]. However, the biological mechanisms driving poor clinical outcomes remain incompletely understood. Therefore, it is necessary to develop prognostic biomarkers and novel therapeutic targets for the treatment and prevention of CRC. In this study, we evaluated Snail and serpinA1 as potential biomarkers in CRC.

Snail is a zinc-finger transcription factor that is known to induce the EMT [23] and was originally shown to be expressed in invasive carcinoma cells [2, 3]. However, some groups have found no evidence of any association between Snail expression in tumors and clinicopathological parameters or overall survival in $\mathrm{CRC}$ in relatively small cohorts $[12,24]$. Thus, in this
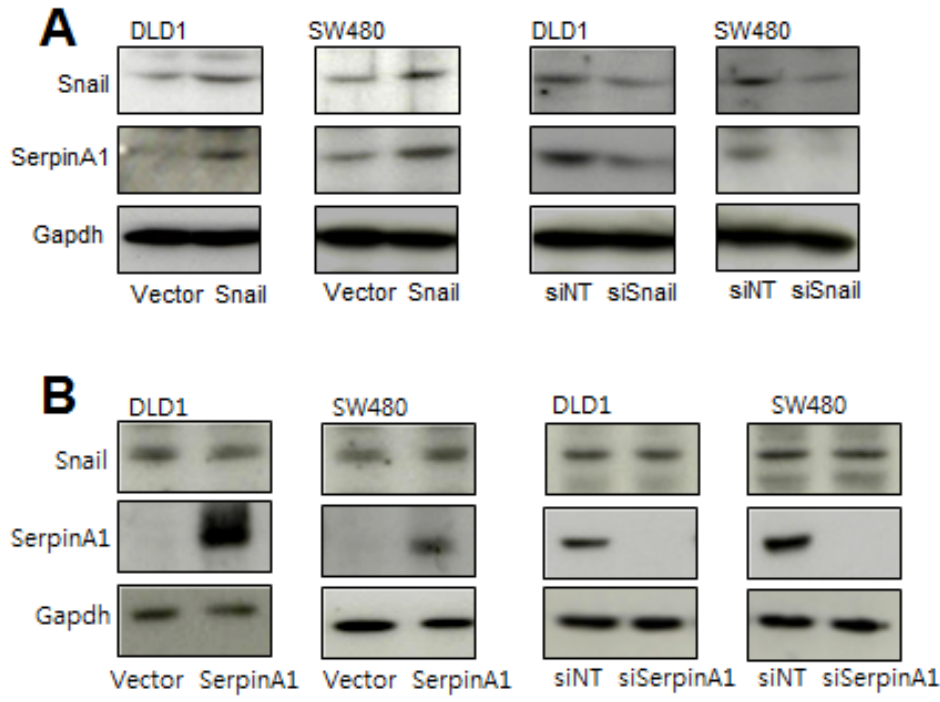
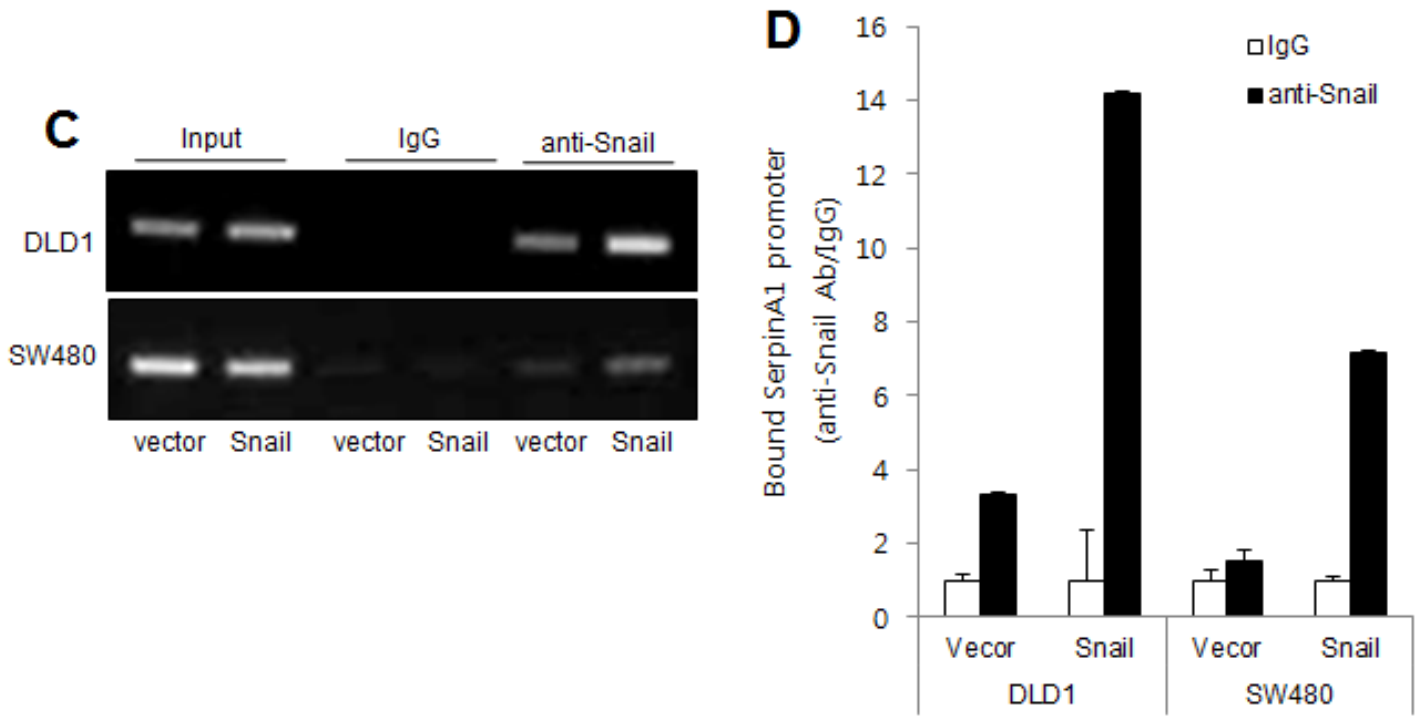

Figure 5: SerpinA1 was regulated by Snail. A. DLD-1 and SW480 cells were transfected with pcDNA-Snail (Snail), control vector pcDNA (vector), Snail siRNA (siSnail), or nontargeting siRNA (siNT), and Snail and serpinA1 protein levels were evaluated by western blot analysis. B. DLD-1 and SW480 cells were transfected with pcDNA-serpinA1 (serpinA1), control vector pcDNA (vector), serpinA1 siRNA (siSerpinA1), or nontargeting siRNA (siNT), and western blot analysis was performed for detection of Snail and SerpinA1 expression. C. DLD-1 and SW480 cells were transfected with pcDNA-Snail (Snail) or control vector pcDNA (vector), and ChIP assays were performed. The presence of the serpinA1 promoter (-516/-4) was verified in immunoprecipitates with either mouse IgG or anti-Snail antibodies, and assay inputs were analyzed using real-time PCR. The samples were loaded on agarose gels. D. Data show promoter enrichment in the antiSnail immunoprecipitate relative to IgG. 
study, we analyzed the clinical significance of Snail expression in CRC development and progression using immunohistochemical analysis in a larger series. Our results showed that Snail was correlated with clinical stage, perineural invasion, lymphovascular emboli, lymph node metastasis, and overall survival in CRC patients. Importantly, further multivariate analyses of our TMA showed that the level of Snail expression was the only significant factor correlated with poor prognosis. Further investigation revealed that overexpression of
Snail increased CRC cell invasion and migration, whereas knockdown of Snail attenuated CRC cell invasion and migration. These results indicated that Snail mediated the invasive and migratory capacity of CRC cells, affecting the metastatic potential of the cells. Interestingly, Snail also induced migration in the other cancer cell lines tested in the present study. Our results are in concordance with other studies, which showed that Snail-expressing cells acquire migratory and invasive properties [25, 26, 27].

Snail was reported to enhance invasiveness and
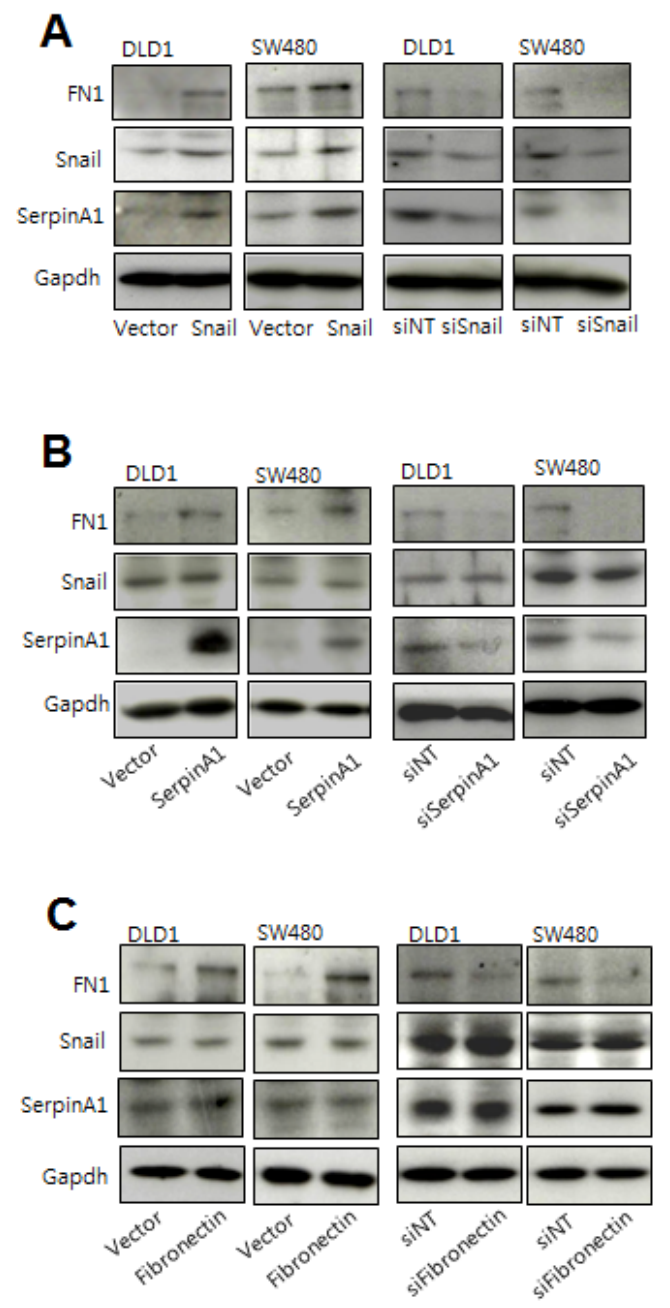
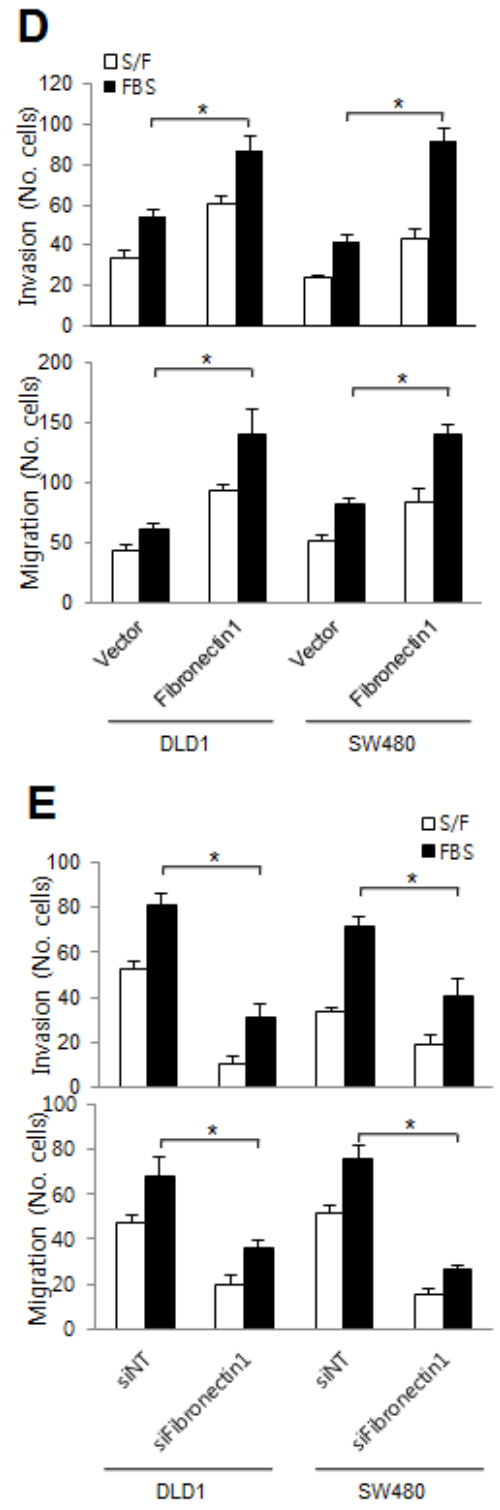

Figure 6: Snail and serpinA1 promoted tumor progression through fibronectin. A. DLD-1 and SW480 cells were transfected with pcDNA-Snail (Snail), control vector pcDNA (vector), Snail siRNA (siSnail), or nontargeting siRNA (siNT), and Snail, serpinA1, and fibronectin protein levels were evaluated by western blot analysis. B. DLD-1 and SW480 cells were transfected with pcDNA-serpinA1 (SerpinA1), control vector pcDNA (vector), serpinA1 siRNA (siSerpinA1), or nontargeting siRNA (siNT), and Snail, serpinA1, and fibronectin protein levels were evaluated by western blot analysis. C. DLD-1 and SW480 cells were transfected with pcDNA-fibronectin (Fibronectin), control vector pcDNA (vector), fibronectin siRNA (siFibronectin), or nontargeting siRNA (siNT), and Snail, serpinA1, and fibronectin protein levels were evaluated by western blot analysis. D., E. Invasion and migration assays were performed using transfected cells. Representative data are shown for cells that invaded (top) and migrated (bottom) in the presence of $1 \%$ FBS. ${ }^{*} P<0.05$. 


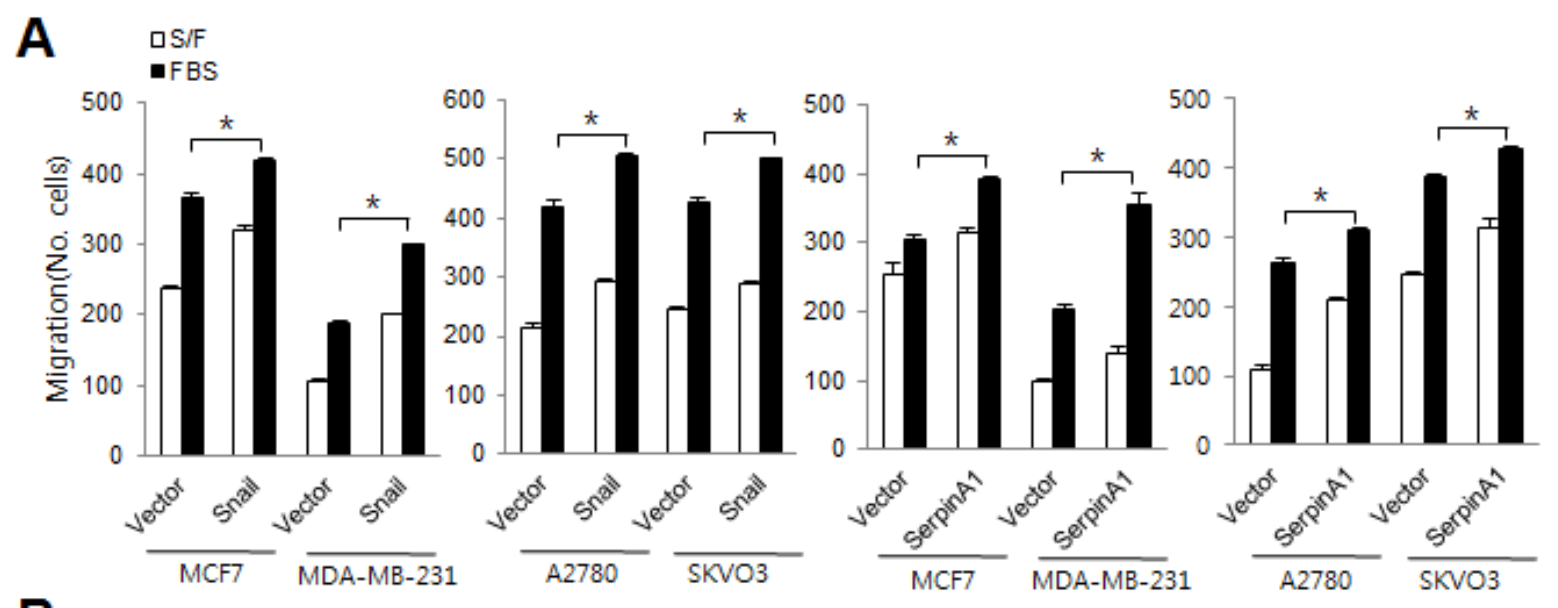

B
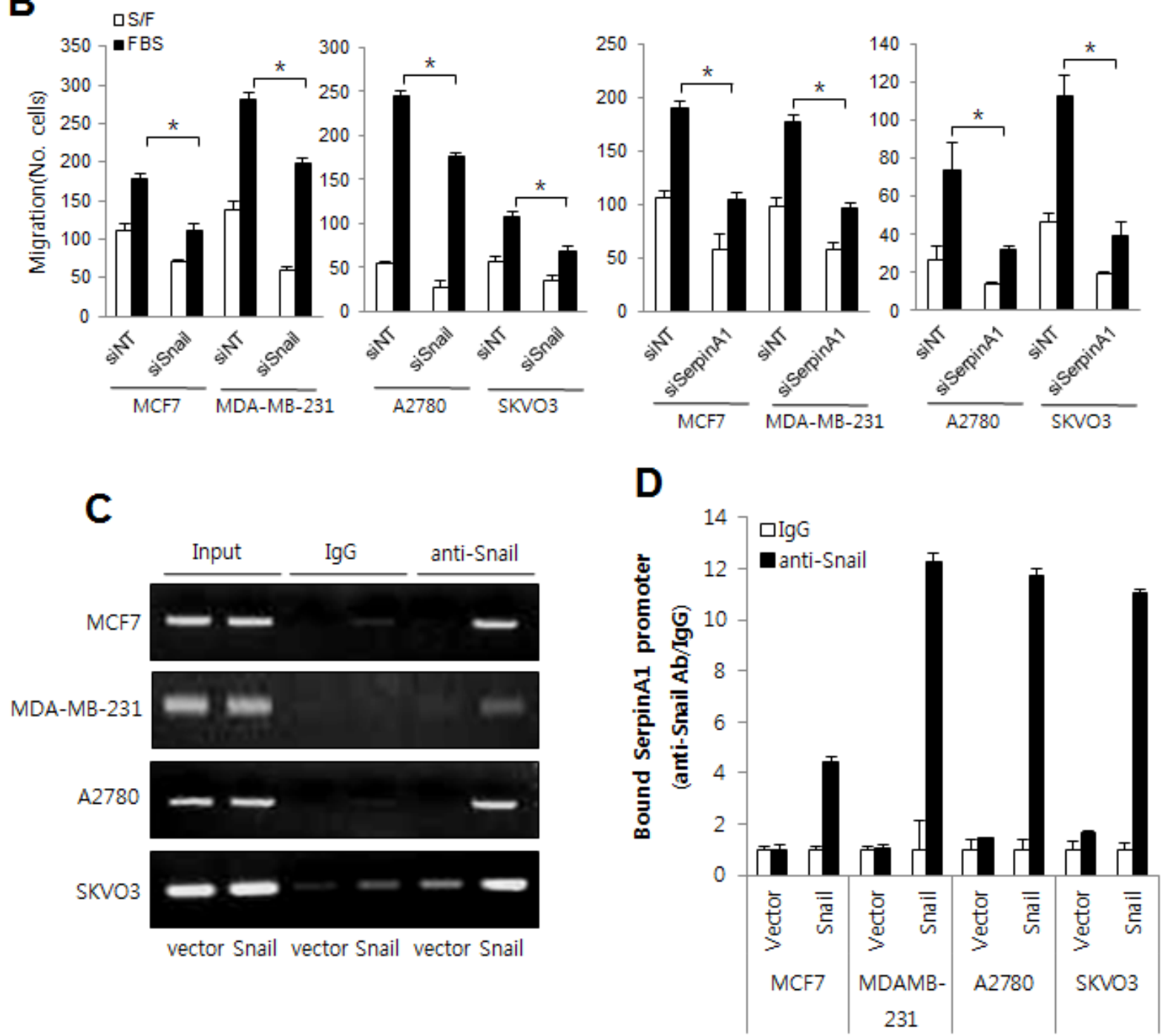

Figure 7: The roles of Snail and serpinA1 in other human cancer cell lines. A., B. MCF7, MDA-MB-231, A2780, and SKVO3 cells were transfected with pcDNA-Snail, pcDNA-serpinA1, Snail siRNA, or serpinA1 siRNA, and migration assays were performed using these cells. Graphs show the number of cells that migrated in the presence or absence of $1 \%$ FBS. ${ }^{*} P<0.05$. C. MCF7, MDAMB-231, A2780, and SKVO3 cells cells were transfected with pcDNA-Snail (Snail) or control vector pcDNA (vector), and ChIP assays were performed. The presence of the serpinA1 promoter (-516/-4) was verified in immunoprecipitates with either mouse IgG or anti-Snail antibodies, and assay inputs were analyzed using real-time PCR. The samples were loaded on agarose gels. D. Data shows promoter enrichment in the anti-Snail immunoprecipitate relative to IgG. 
metastasis through direct repression of E-cadherin transcription [25] or upregulation of metalloproteinases, such as MMP-2 and MMP-9, that are involved indirectly in the degradation of the basement membrane and extracellular matrix [26, 27]. In a previous study, we found that Snail regulates the expression of serpinA1 and is involved in gastric cancer progression $[15,20]$. In this study, immunohistochemical analysis revealed that Snail overexpression was correlated with elevated levels of serpinA1 in CRC. Moreover, serpinA1 was upregulated in CRC cells overexpressing Snail and downregulated in CRC cells exhibiting Snail knockdown. Further analysis using ChIP assays revealed that Snail regulated the expression of serpinA1 through directly binding to the serpinA1 promoter not only in CRC cells but also in breast and ovarian cancer cells. These results show a significant relationship between serpinA1 and Snail in cancer cells and improve our understanding of the mechanism through which Snail is involved in tumor progression.

SerpinA1 is a protease inhibitor that functions as a serum trypsin inhibitor [28]. The serum levels of serpinA1 are higher in cancer patients than in healthy patients [29-31]. In addition, serpinA1 is related to the distant metastasis of various cancers, including ovarian, cervical breast, and lung cancers [32-34]. Consistent with this, we found that serpinA1 was significantly correlated with stage and lymph node metastasis in CRC. In addition, increase serpinA1 expression was related to poor prognosis in patients with CRC patients, and serpinA1 signaling regulated CRC cell motility and invasiveness. Further supporting this, serpinA1 has been reported to be associated with metastasis in CRC [35]. Additionally, a previous study showed that the serum levels of serpinA1 are significantly higher in patients with CRC than in healthy subjects [19]. Moreover, we also observed that serpinA1 promoted migration in breast and ovarian cancer cell lines. These results suggested that serpinA1 plays an important role in tumor progression, and our current data provided evidence of the key role of serpinA1 as a regulator of invasion and migration in CRC cells for the first time.

Although the mechanism of serpinA1 in tumor progression is not fully elucidated, it has been shown to promote lung colonization via fibronectin assembly [21]. In this study, we showed that fibronectin expression was regulated by Snail and serpinA1, suggesting that fibronectin functioned downstream of Snail and serpinA1. SerpinA1 may prevent the disruption of cell surfacefibronectin connections by inhibiting chymase activity, thereby mediating the assembly of fibronectin [36, 37]. Snail is also required for fibronectin activation in epithelial cells undergoing the EMT [38]. Thus, we cannot exclude the possibility that Snail regulates fibronectin expression directly. Further studies are required to elucidate the precise mechanisms through which Snail and serpinA1 mediate fibronectin expression.
Functionally, upregulation of fibronectin in CRC cells promoted invasion and migration, while suppression of fibronectin expression blocked cell invasion and migration, highlighting the role of fibronectin in CRC cell invasion and migration. These results are consistent with the many previous works showing that fibronectin contributes to tumor progression in breast, lung, and thyroid cancer through the activation of multiple oncogenic pathways, such as Akt, extracellular signalregulated kinase, and signal transducer and activator of transcription 3 [39-41]. In our study, we identified a novel mechanism through which fibronectin mediated the motility of CRC cells via Snail and serpinA1. Thus, our data provide important insights into the molecular mechanisms and signaling events regulating CRC cell motility and progression.

In summary, we found that Snail and serpinA1 expression were associated with advanced stage, lymph node involvement, and poor prognosis in patients with CRC. We also provided evidence that Snail and serpinA1 induced CRC cell invasion and migration by upregulation of fibronectin, as well as migration of other cancer cells, demonstrating a novel signaling mechanism involved in tumor progression. These results suggested that Snail and serpinA1 may be useful biomarkers in the clinical setting and new therapeutic targets for development of novel therapeutic modalities in cancer management.

\section{MATERIALS AND METHODS}

\section{Cell lines and transfection}

The human cancer cell lines were obtained from the Korean Cell Line Bank (Seoul, South Korea) or ATCC (VA, USA). The human colon cancer cell lines DLD-1 and SW-480 were cultured in RPMI1640 medium. The human breast cancer cell lines MCF7 and MDA-MB-231 and the human ovarian cancer cell lines A2780 and SKVO3 were maintained in DMEM. All media were supplemented with 10\% fetal bovine serum (FBS; Gibco; Thermo Scientific Inc.; PA, USA), $100 \mathrm{U} / \mathrm{mL}$ penicillin, and $100 \mu \mathrm{g} / \mathrm{mL}$ streptomycin (Sigma-Aldrich; MO, USA). All cells were maintained at $37^{\circ} \mathrm{C}$ in an atmosphere containing $5 \% \mathrm{CO}_{2}$.

For overexpression of genes, cells were grown to $60-70 \%$ confluence and transfected with the pcDNA Snail-Myc, pcDNA SerpinA1-Myc vectors or pcDNA fibronectin-Myc vectors (Origene; MD, USA), or with the pcDNA-3.1 vector as a control, with Lipofectamine 2000 (Invitrogen; Life Technologies; NY, USA) according to the manufacturer's protocol. Cells were maintained in complete medium for 24 hours before the assays were performed.

For knockdown of genes, cells were transfected with Snail or serpinA1 smartpool short interfering 
RNA (siRNA) or with non-targeting siRNA as a control (Dharmacon; Thermo Scientific Inc.; PA, USA), using Lipofectamine RNAiMAX reagent (Invitrogen) according to the manufacturer's instructions. The siRNA sequences were as follows: Snail siRNA, 5'-GCGAGCUGCAGGACUCUAA-3', 5'-AAUCGGAAGCCUAACUACA-3', 5'-GUGACUAACUACUGCAAUAA-3', 5'-GAGUAAUGGCUGUCACUUG-3'; serpinA1 SiRNA, 5'-GAACUCACCCACGAUAUCA-3', 5'-GAUGAAGCGUUUAGGCAUG-3', 5'-CCUAUGAUCUGAAGAGCGU-3', 5'-CCAAGAAACAGAUCAACGA-3'; fibronectin SiRNA, 5'-GAACUCACCCACGAUAUCA-3', 5'-GAUGAAGCGUUUAGGCAUG-3', 5'-CCUAUGAUCUGAAGAGCGU-3', 5'-CCAAGAAACAGAUCAACGA-3';

\section{Real time reverse transcription (RT)-PCR analysis}

Total RNA was extracted from gastric cancer cells, using the TRIzol reagent (Invitrogen), following the manufacturer's instructions. RNA was reverse transcribed with SuperScript II (Invitrogen) and cDNA was amplified with each primer and visualized with SYBR Green (Applied Biosystems; Life Technologies; NY, USA), using the fluorescence reader Corbett Rotor-Gene 6000 (Qiagen Inc.; CA, USA). The primers used are the following:: glyceraldehyde 3-phosphate dehydrogenase (GAPDH), 5'-TCCATGACAACTTTGGTATCG-3', 5'-TGTAGCCAAATTCGTTGTCA-3';

Snail, 5'-TCTTCCTCTCCATACCTG-3', 5'-CATAGTTAGTCACACCTCGT-3'; and serpinA1, 5'-GGACACCGAGGAAGAGGA-3', 5'-TCAGGCAGGAAGAAGATGG-3'. The following thermal cycler program was used: denaturation for 30 $\mathrm{s}$ at $95^{\circ} \mathrm{C}$; annealing for $30 \mathrm{~s}$ at $52^{\circ} \mathrm{C}$, depending on the primers used; and extension for $30 \mathrm{~s}$ at $72^{\circ} \mathrm{C}$. The number of PCR cycles was determined for each gene and ranged from 25 to 35. Data were normalized to GAPDH, and mRNA abundance was calculated using the $2^{-\Delta \Delta C T}$ method. The PCR products were confirmed by mobility on gel electrophoresis.

\section{Chromatin immunoprecipitation (ChIP) assays}

ChIP assays were performed using the EZ-ChIP kit (Merck Millipore; Darmstadt, Germany). Cells were cross-linked with $2 \%$ formaldehyde for $10 \mathrm{~min}$ at $37^{\circ} \mathrm{C}$ and washed in ice-cold PBS. Unreacted formaldehyde was quenched with $200 \mathrm{mM}$ glycine, and then cells were washed with PBS and resuspended in SDS lysis buffer containing protease inhibitors. Samples were sheared by sonication on ice, diluted in dilution buffer with inhibitors, and precleared with agarose $\mathrm{G}$ beads. A portion of the supernatant was stored as input, and the remaining supernatant was divided for immunoprecipitation and incubated with anti-Snail (R\&D system; MN, USA) or IgG as a negative control overnight at $4^{\circ} \mathrm{C}$ with agitation. Immune complexes were captured using ProteinA-Sepharose, then washed sequentially in low-salt buffer, high-salt buffer, twice in $\mathrm{LiCl}$ buffer, then twice in TE buffer. Protein was eluted from the beads in fresh elution buffer, cross-linking was reversed overnight at $65^{\circ} \mathrm{C}$ in the presence of $\mathrm{NaCl}$, and then samples were ethanol-precipitated. Following centrifugation, pellets were resuspended in TE buffer and incubated sequentially with $50 \mu \mathrm{g} / \mathrm{mL}$ RNase A (30 $\mathrm{min}$ ) and $100 \mu \mathrm{g} / \mathrm{mL}$ proteinase $\mathrm{K}(1 \mathrm{~h})$. DNA was purified by washing with elution buffer and centrifugation and then finally analyzed by real-time PCR. The primer sequence was 5'-AAAGAGCAGGACCCCAAAT-3' and 5'-TCCACCCGAAGTCTACTTCC-3'.

\section{Cell migration and invasion assays}

Gastric cancer cells were harvested with $0.05 \%$ trypsin containing $0.02 \%$ EDTA (Sigma-Aldrich) and suspended in RPMI medium. For the migration assay, membrane filters ( 8 - $\mu \mathrm{m}$ pore size) in disposable 96-well chemotaxis chambers (Neuro Probe; Gaithersburg, MD, USA) were pre-coated with $5 \mathrm{mg} / \mathrm{mL}$ fibronectin for $4 \mathrm{~h}$ at room temperature. Cells $\left(3 \times 10^{3}\right.$ cells/well $)$ were loaded into the upper chambers, and 1\% FBS was loaded into the lower chamber. After $24 \mathrm{~h}$ of incubation, non-migrating cells were removed from the upper chamber with a cotton swab, and the cells on the lower surface of the insert were stained with Hoechst33342 (Sigma-Aldrich). Migrated cells were counted under a fluorescence microscope at $10 \times$ magnification.

For the invasion assay, $3 \times 10^{4}$ cells/well were seeded in the upper chamber, which was coated with $30 \mu \mathrm{L}$ of Matrigel ( $1 \mathrm{mg} / \mathrm{mL}$ in cold medium; BD Transduction Laboratories; NJ, USA). Serum-free medium containing 1\% FBS or control vehicle was added to the lower chamber. After $24 \mathrm{~h}$ of incubation, non-invading cells were removed from the upper chamber with a cotton swab, and cells on the lower surface of the insert were stained with Hoechst33342 (Sigma-Aldrich). Invasive cells were counted under a fluorescence microscope at $10 \times$ magnification.

\section{Western blot analysis}

Cells were harvested and disrupted in lysis buffer (1\% Triton X-100, 1 mM EGTA, 1 mM EDTA, $10 \mathrm{mM}$ Tris- $\mathrm{HCl}$ at $\mathrm{pH} \mathrm{7.4,} \mathrm{and} \mathrm{protease} \mathrm{inhibitors).} \mathrm{Cell} \mathrm{debris}$ was removed via centrifugation at $10,000 \times g$ for $10 \mathrm{~min}$ at $4^{\circ} \mathrm{C}$. The resulting supernatants were resolved using SDS- 
PAGE and transferred onto nitrocellulose membranes. The membranes were blocked with 5\% non-fat dried milk at room temperature for $30 \mathrm{~min}$ and incubated with anti-Sanil (AF3639; R\&D system), anti-serpinA1 (HPA000927; Sigma-Aldrich), anti-fibronectin (HPA027066; SigmaAldrich) and anti-GAPDH. The membranes were then washed and incubated with horseradish peroxidaseconjugated secondary antibody. Signals were visualized using enhanced chemiluminescence (Amersham; Buckinghamshire, UK).

\section{Immunohistochemistry and analysis of clinicopathological and prognostic significance}

We studied a cohort of 528 CRC patients who received resection of the primary tumor at the Pusan National University Hospital (PNUH) between 2003 and 2008. Standard formalin-fixed and paraffin-embedded sections were obtained from the Department of Pathology and the National Biobank of Korea, PNUH. All samples from the National Biobank of Korea were obtained with informed consent under institutional review boardapproved protocols.

Methods of immunohistochemistry have previously been described for Snail [20] and SerpinA1 [15]. Snail staining was considered positive when nuclear staining was detectable and graded as $<75 \%$ and $\geq 75 \%$ positivity, which has been described in our previous study [20]. SerpinA1 staining in tumor cells was considered positive. SerpinA1 immunostaining was graded as follows: negative (-) for no stainig, $(+)$ for any staining, $(++)$ for strong staining.

Clinicopathological features were analyzed for differences in Snail or serpinA1 expression using the Student's $t$-test, the $\chi^{2}$ test, or Fisher's exact test. The relationships between expression of Snail and serpinA1 were assessed with a Spearman rank correlation coefficient. Cumulative survival plots were obtained using the Kaplan-Meier method, and significance was compared using the log-rank test. Statistical significance was set at $P$ $<0.05$. Multivariate analyses were carried out using Cox proportional hazards regression. Statistical calculations were performed using SSPS version 10.0 for Windows (SPSS Inc.; Chicago, IL, USA).

\section{ACKNOWLEDGMENTS}

The biospecimens for this study were provided by the Pusan National University Hospital or by members of the National Biobank of Korea, which is supported by the Ministry of Health, Welfare, and Family Affairs. This study was supported by a grant (0920050) from the National R\&D Program for Cancer Control, Ministry for Health, Welfare and Family Affairs, Republic of Korea.

\section{CONFLICTS OF INTEREST}

No potential conflicts of interest were disclosed.

\section{REFERENCES}

1. Thiery JP. Epithelial-mesenchymal transitions in tumour progression. Nat Rev Cancer 2002;2:442-54.

2. Cano A, Pe'rez-Moreno MA, Rodrigo I, Locascio A, Blanco MJ, del Barrio MG, Portillo F, Nieto MA. The transcription factor Snail controls epithelial mesenchymal transitions by repressing E-cadherin expression. Nat Cell Bio 2000;2:76-83.

3. Batlle E, Sancho E, Franci C, Domı'nguez D, Monfar M, Baulida J, Garc1'a De, Herreros A. The transcription factor Snail is a repressor of E-cadherin gene expression in epithelial tumour cells. Nat Cell Biol 2000;2:84-9.

4. Blanco MJ, Moreno-Bueno G, Sarrio D, Locascio A, Cano A, Palacios J, Nieto MA. Correlation of Snail expression with histological grade and lymph node status in breast carcinomas. Oncogene 2002;21: 3241-6.

5. Martin TA, Goyal A, Watkins G, Jiang WG. Expression of the transcription factors snail, slug, and twist and their clinical significance in human breast cancer. Ann Surg Oncol 2005;12:488-96.

6. Elloul S, Elstrand MB, Nesland JM, Trope CG, Kvalheim G, Goldberg I, Reich R, Davidson B. Snail, Slug, and Smad-interacting protein 1 as novel parameters of disease aggressiveness in metastatic ovarian and breast carcinoma. Cancer 2005;103: 1631-43.

7. Emadi BM, Soheili ZS, Schmitz I, Sameie S, Schulz WA. Snail regulates cell survival and inhibits cellular senescence in human metastatic prostate cancer cell lines. Cell Biol Toxicol 2010;26:553-67.

8. Sugimachi K, Tanaka S, Kameyama T, Taguchi K, Aishima S, Shimada M, Sugimachi K, Tsuneyoshi M. Transcriptional repressor snail and progression of human hepatocellular carcinoma. Clin Cancer Res 2003;9:265764.

9. Kuphal S, Palm HG, Poser I, Bosserhoff AK. Snailregulated genes in malignant melanoma. Melanoma Res 2005;15:305-13.

10. Castro Alves C, Rosivatz E, Schott C, Hollweck R, Becker I, Sarbia M, Carneiro F, Becker KF. Slug is overexpressed in gastric carcinomas and may act synergistically with SIP1 and Snail in the downregulation of E-cadherin. J Pathol 2007;211:507-15.

11. Roy HK, Smyrk TC, Koetsier J, Victor TA, Wali RK. The transcriptional repressor SNAIL is overexpressed in human colon cancer. Dig Dis Sci 2005;50:42-6.

12. Kroepil F, Fluegen G, Vallböhmer D, Baldus SE, Dizdar L, Raffel AM, Hafner D, Stoecklein NH, Knoefel WT. Snaill expression in colorectal cancer and its correlation with clinical and pathological parameters. BMC Cancer. 
2013;13:145.

13. Backlund MG, Mann JR, Holla VR, Shi Q, Daikoku T, Dey SK, DuBois RN. Repression of 15-hydroxy prostaglandin dehydrogenase involves histon deacetylase 2 and snail in colorectal cancer. Cancer Res. 2008;68:9331-1.

14. Pena C, Garc1'a JM, Silva J, Garc1'a V, Rodri'guez R, Alonso I, Milla'n I, Salas C, de Herreros AG, Muñoz A, Bonilla F. E-cadherin and vitamin $\mathrm{D}$ receptor regulation by SNAIL and ZEB1 in colon cancer: clinicopathological correlations. Hum Mol Genet 2005; 14:3361-70.

15. Kwon CH, Park HJ, Lee JR, Kim HK, Jeon TY, Jo HJ, Kim DH, Park DY. SerpinA1 peptidase inhibitor clade A member 1 is a biomarker of poor prognosis in gastric cancer. Br J Cancer 2014 (in press).

16. Higashiyama M, Doi O, Kodama K, Yokouchi H, Tateishi R. An evaluation of the prognostic significance of alpha-1antitrypsin expression in adenocarcinomas of the lung: an immunohistochemical analysis. Br J Cancer 1992;65:300-2.

17. Tahara E, Ito H, Taniyama K, Yokozaki H, Hata J. Alpha 1-antitrypsin, alpha 1-antichymotrypsin, and alpha 2-macroglobulin in human gastric carcinomas: a retrospective immunohistochemical study. Hum Pathol 1984;15:957-64.

18. Karashima S, Kataoka H, Itoh H, Maruyama R, Koono M. Prognostic significance of alpha-1-antitrypsin in early stage of colorectal carcinomas. Int J Cancer 1990;45:244-50.

19. Bujanda L, Sarasqueta C, Cosme A, Hijona E, EnríquezNavascués JM, Placer C, Villarreal E, Herreros-Villanueva M, Giraldez MD, Gironella M, Balaguer F, Castells A. Evaluation of alpha 1-antitrypsin and the levels of mRNA expression of matrix metalloproteinase 7 , urokinase type plasminogen activator receptor and COX-2 for the diagnosis of colorectal cancer. PLoS One 2013;8:e51810.

20. Shin NR, Jeong EH, Choi CI, Moon HJ, Kwon CH, Chu IS, Kim GH, Jeon TY, Kim DH, Lee JH, Park do Y. Overexpression of Snail is associated with lymph node metastasis and poor prognosis in patients with gastric cancer. BMC cancer 2012;12:521.

21. Chang YH, Lee SH, Liao IC, Huang SH, Cheng HC, Liao PC. Secretomic analysis identifies alpha-1 antitrypsin (A1AT) as a required protein in cancer cell migration, invasion, and pericellular fibronictin assembly for facilitating lung colonization of lung adenocarcinoma cells. Mol Cell Proteomics 2012;11:1320-39.

22. Koh SJ, Kim JS. The reasons for the increased incidence of colorectal cancer in Korea. Kor J Med 2010;79:97-103.

23. Nieto MA. The snail superfamily of zinc-finger transcription factors. Nat Rev Mol Cell Biol 2002;3:155-66

24. Francí C, Gallén M, Alameda F, Baró T, Iglesias M, Virtanen I, García de Herreros A. Snail1 protein in the stroma as a new putative prognosis marker for colon tumours. PLoS One 2009;4:e5595.

25. Barrallo-Gimeno A, Nieto MA. The Snail genes as inducers of cell movement and survival: implications in development and cancer. Development 2005; 132:3151-61.

26. Yokoyama K, Kamata N, Fujimoto R, Tsutsumi S, Tomonari M, Taki M, Hosokawa H, Nagayama M. Increased invasion and matrix metalloproteinase-2 expression by Snail-induced mesenchymal transition in squamous cell carcinomas. Int J Oncol 2003;22: 891-8.

27. Jorda M, Olmeda D, Vinyals A, Valero E, Cubillo E, Llorens A, Cano A, Fabra A. Upregulation of MMP-9 in MDCK epithelial cell line in response to expression of the Snail transcription factor. J Cell Sci 2005;118:3371-85.

28. Gettins PG. Serpin structure, mechanism, and function. Chem Rev 2002;102: 4751-4804.

29. El-Akawi ZJ, Al-Hindawi FK, Bashir, NA. Alpha-1 antitrypsin (alpha1-AT) plasma levels in lung, prostate and breast cancer patients. Neuroendocrinol Lett 2008;29:482484

30. El-Akawi ZJ, Abu-Awad AM, Sharara AM, Khader Y. The importance of alpha-1 antitrypsin (alpha1-AT) and neopterin serum levels in the evaluation of non-small cell lung and prostate cancer patients. Neuroendocrinol Lett 2010;31:113-116.

31. Comunale MA, Rodemich-Betesh L, Hafner J, Wang M, Norton P, Di Bisceglie AM, Block T, Mehta A. Linkage specific fucosylation of alpha-1-antitrypsin in liver cirrhosis and cancer patients: implications for a biomarker of hepatocellular carcinoma. PLoS One 2010;5:e12419.

32. Normandin $\mathrm{K}, \mathrm{Pe}^{\prime}$ ant $\mathrm{B}$, Le Page $\mathrm{C}$, de Ladurantaye $\mathrm{M}$, Ouellet V, Tonin PN, Provencher DM, Mes-Masson AM. Protease inhibitor SERPINA1 expression in epithelial ovarian cancer. Clin Exp Metastasis 2010;27:55-69.

33. Woz' niak B, Mila-Kierzenkowska C, WoY niak A, Drewa G, Sopon' ska M, Drewa T, Krzyzyn' ska-Malinowska E, Makarewicz R, Kowalski T, Szmytkowska K. The effect of combined therapy on activity of cathepsin D and alpha1 -antitrypsin in the blood serum of women with cervical cancer. Eur J Gynaecol Oncol 2008;29:617-619.

34. Mbeunkui F, Metge BJ, Shevde LA, Pannell LK. Identification of differentially secreted biomarkers using LC-MS/MS in isogenic cell lines representing a progression of breast cancer. Proteome Res 2007;6:2993-3002.

35. Koh KH, Rhee H, Kang HJ, Yang E, You KT, Lee H, Min BS, Kim NK, Nam SW, Kim H. Differential gene expression profiles of metastases in paired primary and metastatic colorectal carcinomas. Oncology 2008;75:92101.

36. Aziz MH, Hafeez BB, Sand JM, Pierce DB, Aziz SW, Dreckschmidt NE, Verma AK. Protein kinase Cvarepsilon mediates Stat3Ser727 phosphorylation, Stat3-regulated gene expression, and cell invasion in various human cancer cell lines through integration with MAPK cascade (RAF-1, MEK1/2, and ERK1/2). Oncogene 2010;29:3100-9.

37. Morgan K, Marsters P, Morley S, van Gent D, Hejazi A, Backx M, Thorpe ER, Kalsheker N. Oncostatin M induced alpha1 - antitrypsin (AAT) gene expression in Hep G2 cells 
is mediated by a 3'enhancer. Biochem J 2002;365:555-560.

38. Stanisavljevic J, Porta-de-la-Riva M, Batlle R, de Herreros AG, Baulida J. The p65 subunit of NF- $\mathrm{kB}$ and PARP1 assist Snaill in activating fibronectin transcription. J Cell Sci. 2011;124:4161-71.

39. Balanis N, Wendt MK, Schiemann BJ, Wang Z, Schiemann WP, Carlin CR. Epithelial to mesenchymal transition promotes breast cancer progression via a fibronectindependent STAT3 signaling pathway. J Biol Chem 2013;288:17954-67.

40. Sun X, Fa P, Cui Z, Xia Y, Sun L, Li Z, Tang A, Gui Y, Cai Z. The EDA-containing cellular fibronectin induces epithelial mesenchymal transition in lung cancer cells through integrin $\alpha 9 \beta 1$-mediated activation of PI3K/AKT and Erk1/2. Carcinogenesis 2014;35:184-91.

41. Liu W, Cheng S, Asa SL, Ezzat S. The melanoma associated antigen A3 mediates fibronectin-controlled cancer progression and metastasis. Cancer Res 2008;68:8104-12. 